\title{
AN APPLICATION FOR THE DOCTORS+
}

\author{
Vishwam Shukla \\ Department of CSE \\ MIT ADT University, Pune, Maharashtra, India
}

\begin{abstract}
Doctors+ is an application intended with the focal goal of creating a convenient and reliable health care system. It holds data integrity, effective communication, reliable storage and relay of information among many of its central objectives. Further integration with intelligent systems allows it to provide an interactive and predictive interface for the stakeholders involved.
\end{abstract}

Keywords - Out-patient department, In-patient Department, Pharmacy, Emergency Department.

\section{INTRODUCTION}

The management and communication of information in hospital environments is a crucial aspect that needs to be monitored. Patient records need to be constantly accessed by doctors, nurses, ward-in charges and other employees of the hospital. Doctors+ is an application that has been developed keeping the requirements of a hospital environment in mind. Intending to provide a working system that is up to $90 \%$ paperless, it tackles a variety of issues regarding effective communication to create a convenient and reliable system. The system ensures thorough protection of data while also providing a robust and reliable backup facility.

\section{FUNCTIONALITY}

The user interface is designed to manage patient details and refer them to hospital employees with ease. The application acts as a singular hub where all employees can access, update and track details of prior patients or those that have made the first visit. Various modules interconnect the different departments concerned and can be understood as follows: The first interface of the app displays the 6 categories for accessing and relaying data within the application. This includes Doctors, Receptionists, Nurse, Pharmacists, Emergency and Admin. The Login Portal offers access to each of the medical staff involved to access and update data as and when required.

The main home page stores all the schedules and appointments to keep track of all the dates, times and other specific details The accounts page is a profile page for each party involved where they can update personal contact information and other information including a profile picture for easy reference. A drawer slider display organizes some functions and details for cross consultancies, emergencies and other details of patients. The Out-Patient Department can be updated with the patient's referral information. It provides an inter-connection with

\author{
Shreeya Parikh \\ Department of CSE \\ MIT ADT University, Pune, Maharashtra, India
}

different departments acting like a reception for the OPD and IPD departments. Many other tasks like managing leave applications, making IPD requests, etc can be carried out through this dashboard. The prior medical history of the patients can be stored, updated and accessed on each consecutive visit. Integration with the pharmacy updates on the requirements of medicine and equipment as per the current patient's needs. The current iteration of the application focuses on engaging AI to increase the interaction between the different stakeholders.

\section{A. Chatbot -}

An IBM Watson Chatbot in integration with the interface provides a fast channel for messaging across platforms. The user-created chatbot can seamlessly connect to web environments, messaging channels and social networks, thus, making scaling easy. A virtual agent can also be used to provide automated suggestions to the users at the customer end.

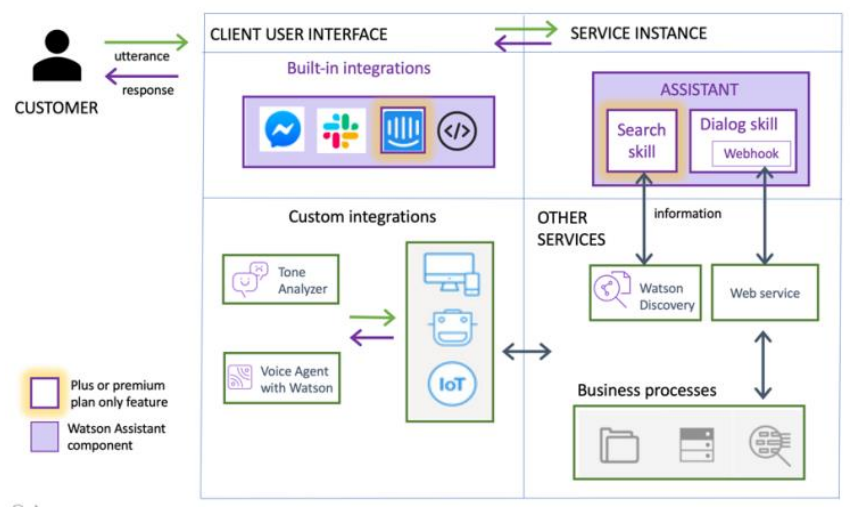

Fig. 1. Chatbot

The virtual agent provides a cognitive, conversational, selfservice experience that prompts answers to the users and takes necessary actions. It is customized to fit the requirements of hospital requirements and deep analytics is used to understand the changing needs of the patients. The users can interact with the virtual assistant through multiple integration points. The assistant takes the user's input and routes it to the appropriate dialogue skills. The dialogue skill decodes the user's input and then directs the conversation's course. The dialogue gathers any information it needs to respond to or perform a transaction on the user's behalf. Any questions that cannot be answered by the dialogue skill are sent to the search skill, which finds relevant 


\section{International Journal of Engineering Applied Sciences and Technology, 2021 Vol. 6, Issue 3, ISSN No. 2455-2143, Pages 106-110 \\ Published Online July 2021 in IJEAST (http://www.ijeast.com)}

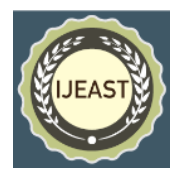

answers by searching the company knowledge bases that you configure for the purpose.

\section{B. Predictive Analysis -}

The application provides predictive analysis based on a predetermined set of diagnostics and prescriptions which can be made available through AI intervention. Prediction and prevention are inextricably linked, probably nowhere more so than in community health management. Organizations that can identify people at high risk of acquiring chronic diseases early in the disease's progression have the best opportunity of helping patients avoid costly and difficult-to-treat long-term health problems. Using lab testing, biometric data claims data, patientgenerated health data, and social determinants of health to create risk scores might help healthcare practitioners figure out who would benefit from improved treatments or wellness programmes. While still in the hospital, patients face several potential threats to their wellbeing, including the development of sepsis, the acquisition of a hard-to-treat infection, or a sudden downturn due to their existing clinical conditions. Data analytics can help providers react as quickly as possible to changes in a patient's vitals, and may be able to identify an upcoming deterioration before symptoms manifest themselves to the naked eye.

\section{Natural Language Processing -}

NLP entails a field of machine learning to analyze, understand and manipulate the generation of human-like language. This is the basic principle of the working of chatbots. It involves concepts regarding "intents" and" entities." Intents represent the intention of a user when he/she addresses a chatbot. Entities are words that put intentions into context and give them meaning. A chatbot with the IBM Watson Assistant platform employs "dialogue" and "digressions". Dialogue refers to the dialogue flow which the chatbot need to follow to have a structured speech. Digressions refer to the conditions which allow the user of the chat-bot to jump from one intent to another and return to the former one if intended. Those represent a set of rules that allow the chatbot to change context without losing the sense of the speech.

\section{K-Fold Cross-Validation -}

$\mathrm{K}$-fold Cross Validation is an algorithm mainly used in machine learning related problems where the main goal is to predict something and/or to estimate the reliability of a predictive model. It is most commonly used in Supervised Learning models, in which a data set with known target labels is provided and training is performed on it (training data-set). It is also given a data-set of unlabeled data, against which the model is tested (testing set). The purpose of cross-validation is to test the model's ability to predict new data that were not used in estimating it, to flag problems like over-fitting and to provide information on how the model will generalise after it is implemented in production The original sample is randomly partitioned into k equal sized sub-samples of the k sub-samples, a single subsample is maintained as validation data for testing the model, while the remaining $\mathrm{k} 1$ subsamples are utilised as training data to test and validate the proposed system. The $\mathrm{k}$ number of folds' results can then be averaged to get a single estimation. This method has the benefit of repeated random subsampling in that all observations are used for both training and validation, and each representation is used only once for validation. Cross-validation is a technique to evaluate predictive models by partitioning the original sample into a training set to train the model, and a test set to evaluate it. In kfold cross-validation, the original sample is randomly partitioned into $\mathrm{k}$ equal size subsamples. One of the $\mathrm{k}$ subsamples is kept as validation data for testing the model, while the remaining $\mathrm{k}-1$ subsamples are used as training data. The cross-validation procedure is then repeated $\mathrm{k}$ times (the folds), with each of the $\mathrm{k}$ subsamples serving as validation data exactly once. The $\mathrm{k}$ results from the folds can then be averaged (or otherwise combined) to produce a single estimation. The advantage of this method is that all observations are used for both training and validation, and each observation is used for validation exactly once. Stratified k-fold cross-validation is commonly used for classification issues, in which the folds are chosen so that each fold has nearly the same proportions of class labels. In repeated cross-validation, the cross-validation procedure is repeated $\mathrm{n}$ times, yielding $\mathrm{n}$ random partitions of the original sample. To obtain a single estimate, the $\mathrm{n}$ findings are again averaged (or otherwise combined). Given the number of folds and repeats, OpenML generates train-test splits so that various users can evaluate their models using the same splits. For classification difficulties, stratification is used by default (unless otherwise specified). The splits are provided as an ARFF file with the row id, fold number, repeat number, and class as part of the task description (TRAIN or TEST). The fold and repeat number of the test instance should be labelled on the uploaded predictions so that the results may be correctly analyzed and aggregated. Both the per fold/repeat results and the aggregated scores are saved in OpenML.

\section{E. Real-time Ambulance tracking -}

The application is integrated with a real-time ambulance tracking system. The ambulance page allows filling in the details of the patient in need of an ambulance. The details of the patient can be relayed and necessary arrangements can be made to accompany the ambulance. In addition to this, it facilitates real-time tracking for the ambulance to have prompt updates of the location of the ambulance. It also helps, additionally, to find the location where the ambulance is demanded on the map for a fast and quick response time. The real-time GPS tracking system has several advantages to not only provide the least minimum time but also to keep track of the number of ambulances employed at the moment and to track which ones are available for allotment. When dispatching emergency 


\section{International Journal of Engineering Applied Sciences and Technology, 2021 Vol. 6, Issue 3, ISSN No. 2455-2143, Pages 106-110 \\ Published Online July 2021 in IJEAST (http://www.ijeast.com)}

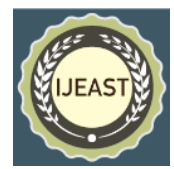

services to the scene of an accident or other dangerous circumstance, dispatchers in charge of managing fleet tracking activity of ambulances must make immediate decisions. They must take every precaution to ensure that the nearest medical care vehicles arrive at the scene of any incident as soon as feasible and that those vehicles use the shortest route possible. Ambulance GPS car trackers can help in this situation. Ambulance businesses and hospital dispatchers can have complete control over ambulance fleet activities by installing a live GPS tracker on an ambulance as a vehicle management solution. This lets the dispatcher or company see where each ambulance is in real-time, as well as allowing fleet supervisors to analyse past driving routes at any time. Because real-time GPS trackers for ambulances provide end-users with lifelong access to previous driving routes, this is the case. Fleet managers may check where an ambulance was earlier in the day, last week, last month, a year ago, or even further back with this lifetime access to historical driving records. Nothing is more vital than getting the right medical assistance from one point to another as quickly as possible in a life-threatening scenario. Ambulance GPS trackers are assisting in reducing ambulance arrival times and so saving lives. The data from the ambulance GPS tracker is safely stored on business servers, from which the firm can access historical driving activity at any time. That is a point on which everyone can agree. The data from the ambulance GPS tracker is securely saved on company servers, allowing the company to view historical driving activities at any time.

\section{F. Real-time Ambulance tracking -}

The application is integrated with a real-time ambulance tracking system. The ambulance page allows filling in the details of the patient in need of an ambulance. The details of the patient can be relayed and necessary arrangements can be made to accompany the ambulance. In addition to this, it facilitates real-time tracking for the ambulance to have prompt updates of the location of the ambulance. It also helps, additionally, to find the location where the ambulance is demanded on the map for a fast and quick response time. The real-time GPS tracking system has several advantages to not only provide the least minimum time but also to keep track of the number of ambulances employed at the moment and to track which ones are available for allotment. When dispatching emergency services to the scene of an accident or other dangerous circumstance, dispatchers in charge of managing fleet tracking activity of ambulances must make immediate decisions. They must take every precaution to ensure that the nearest medical care vehicles arrive at the scene of any incident as soon as feasible and that those vehicles use the shortest route possible. Ambulance GPS car trackers can help in this situation. Ambulance businesses and hospital dispatchers can have complete control over ambulance fleet activities by installing a live GPS tracker on an ambulance as a vehicle management solution. This lets the dispatcher or company see where each ambulance is in real-time, as well as allowing fleet supervisors to analyse past driving routes at any time. Because real-time GPS trackers for ambulances provide end-users with lifelong access to previous driving routes, this is the case. Fleet managers may check where an ambulance was earlier in the day, last week, last month, a year ago, or even further back with this lifetime access to historical driving records. Nothing is more vital than getting the right medical assistance from one point to another as quickly as possible in a life-threatening scenario. Ambulance GPS trackers are assisting in reducing ambulance arrival times and so saving lives. The data from the ambulance GPS tracker is safely stored on business servers, from which the firm can access historical driving activity at any time. That is a point on which everyone can agree. The data from the ambulance GPS tracker is securely saved on company servers, allowing the company to view historical driving activities at any time.

\section{G. Text Recognition -}

Optical Character Recognition is the algorithm used for text recognition. The application uses text recognition for scanning text from medicine strips, brochures, etc for a direct prescription. The algorithm enables conversion of typed, handwritten, or printed text images into machine-encoded text via electronic means. It recognises printed text in paper documents, handwritten characters, and text elements in the real world, such as licence plate numbers, street signs, and street numbers. Pattern matching, pattern recognition, and picture correlation are all used in traditional OCR methods. In a typical use case, like as a document scanner, these approaches can distinguish words and phrases with a high level of accuracy. Look for white pixel rows with black pixels in between to identify a line of text. We can discern where a character begins and finishes in the same way. The character's picture is then converted into a binary matrix, with white pixels equaling 0 s and black pixels equaling 1 . The distance from the matrix's centre to the farthest 1 may then be calculated using the distance formula. Then we make a circle with that radius and divide it into smaller portions. At this point, the algorithm compares each subsection to a database of matrices representing characters in various fonts in order to determine the character with which it shares the most statistical similarities. By doing so for each line and character, it makes converting printed media to digital a breeze.

\section{H. Fall Detection -}

The user of a medical alert system with fall detection can seek help without touching the call button. If the user falls, these systems will immediately activate the sensor. By detecting rapid changes in body motions, fall warning detectors can determine whether a user has fallen. The device can assess a person's smoothness with which movements are accelerated, as well as body position and physical activity. If these variables are in the danger zone and a fall occurs, the device will automatically activate an emergency fall alert and call for help from emergency response agents. Automatic fall detection 


\section{International Journal of Engineering Applied Sciences and Technology, 2021 \\ Vol. 6, Issue 3, ISSN No. 2455-2143, Pages 106-110 \\ Published Online July 2021 in IJEAST (http://www.ijeast.com)}

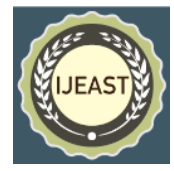

technology accurately detects the majority of frequent motions associated with falls. A medical alert device, when used appropriately, can promptly summon emergency responders. The agents can assess the problem via the device's two-way speaker and will remain on the line with the user until help arrives. The Bayes Theorem establishes a consistent method for estimating conditional probability. It's a deceptively basic formula that provides an easy-to-use solution for situations where our intuition frequently fails. Thinking about the meaning of the elements in the equation and applying the calculation many times in a variety of real-world situations is the best method to build an understanding of Bayes Theorem. The Bayes Theorem is a mathematical formula that is used to calculate the probability of a Conditional probability is the likelihood of one event based on the occurrence of another event, and it is often described in terms of occurrences A and B from two dependent random variables, such as $\mathrm{X}$ and $\mathrm{Y}$.

$\mathrm{P}(\mathrm{A}$ given $\mathrm{B})$ or $\mathrm{P}(\mathrm{A} \mid \mathrm{B})$ are examples of conditional probability, which is the probability of one (or more) events based on the existence of another event.

The joint probability can be used to compute the conditional probability, for example:

$$
\text { - } \mathrm{P}(\mathrm{A} \mid \mathrm{B})=\mathrm{P}(\mathrm{A} \text { and } \mathrm{B}) / \mathrm{P}(\mathrm{B})
$$

For example, the conditional probability is not symmetrical:

$$
\text { - } \quad \mathrm{P}(\mathrm{A} \mid \mathrm{B}) !=\mathrm{P}(\mathrm{B} \mid \mathrm{A})
$$

This is known as the Bayes Theorem, after Reverend Thomas Bayes, and it goes like this:

$$
\text { - } \quad \mathrm{P}(\mathrm{A} \mid \mathrm{B})=\mathrm{P}(\mathrm{B} \mid \mathrm{A}) * \mathrm{P}(\mathrm{A}) / \mathrm{P}(\mathrm{B})
$$

The Bayes Theorem is a systematic approach to computing a conditional probability that can be used instead of the joint probability.

\section{Face Recognition -}

The motion recognition system is classified into 3 steps: face location determination, emotion classification and feature extraction The face is located using a face detection technique. After this step, The face area is processed using the development of the face along with image processing techniques in order to recognise the feature locations. Distances between feature points are calculated from the extracted feature points and used as input to the neural network to categorise the emotion. In this work, systems will efficiently identify the six universal emotions from 2D colour face images. The effort has been restricted to the universal emotions since classification and identification of other marginal emotions is problematic. The system is divided into three stages: determining the location of the face, extracting features, and classifying emotions. Two face detection algorithms are implemented for the face location determination stage. Some feature points and critical features such as eyebrows, mouth and eyes are extracted to identify the emotion of the faces. The important aspects of the eyes, lips, and brows are discovered, and their feature points are extracted to recognise the emotion. By using a corner point detection algorithm, feature points are extracted from the selected feature regions. After feature extraction is performed a neural network approach is used to identify the emotions enclosed within the face. Face recognition accuracy was increased by classifying unique vectors learned from a set of generic samples. The differentiated vectors on behalf of intrasubject and Inter-subject variations are learned using similarities between pairs of general samples, which are then utilised to categorise fresh intra-subject and inter-subject pairs from the training and data sets. Then, using a voting mechanism, the obtained classification is used to detect faces by combining it with eigenface's communication capacity. The Viola-Jones algorithm has the following properties that make it an excellent detection algorithm: 1. Reliable - has a high detection rate (true-positive rate) and a low false-positive rate at all times. 2. Real-time - For practical applications, at least 2 frames per second must be processed. 3. Only face detection (not recognition) - this detection step differentiates a human face from the image background of a picture. This is the initial step in the process of recognition. Due to face variables such as size, angle, and posture, detecting the human face is a tough process.

\section{J. Emergency Management -}

The app enables a robust emergency management system. This includes effective communication between the ambulance and sites where they are required. The emergency management department focuses on organizing resources such as ambulance availability and location, as well as hospital ED and intensive care unit bed capacities and other details. Historically, correct information has been reliant on frequently unreliable telephonic and two-way radio contact between ambulances, hospitals, and incident command centres. The app's goal is to provide precise, real-time data from the disaster scene, which can help EDs predict workloads from ambulance arrivals and ambulatory patients. This information can be critical in preparing for interventions, such as decontamination prior to arrival, as well as coordinating employees and resources within the hospital. This technology can help with field-to-ED care integration, especially in terms of patient information transfer. Patient medical information and field treatment, including decontamination status, might be downloaded by ED staff 
Published Online July 2021 in IJEAST (http://www.ijeast.com)

through patient tracking devices linked to a central database of patient data and information.

\section{CONCLUSION}

As a result, Doctors+ can be utilised to provide paperless hospitals up to $90 \%$ of the time, resulting in superior patient care.

\section{REFERENCES}

[1] D. Griffiths, D. Griffiths, Head First Android Development, 2 (2017) 